\title{
HÁBITOS ALIMENTARES EM DISTINTOS PERÍODOS PANDÉMICOS AO LONGO DA HISTÓRIA: UMA REVISÃO NARRATIVA DA LITERATURA
}

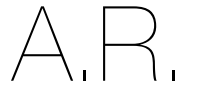
ARTIGO DE REVISÃO

\footnotetext{
${ }^{1}$ Centro de Investigação Interdisciplinar Egas Moniz do Instituto Universitário Egas Moniz,

Campus Universitário Quinta da Granja, 2829-511 Monte de Caparica, Portugal

2 Centro de Investigação em Qualidade de Vida do Instituto Politécnico de Santarém / Instituto Politécnico de Leiria, Avenida Dr. Mário Soares, n. ${ }^{\circ} 110$,

2040-413 Rio Maior,

Portugal

${ }^{3}$ Escola Superior Agrária, Instituto Politécnico de Santarém,

Quinta do Galinheiro - S.

Pedro,

2001-904 Santarém, Portugal
}

*Endereço para correspondência:

Rui Jorge

Escola Superior Agrária do Instituto Politécnico de Santarém

Quinta do Galinheiro - S. Pedro, 2001-904 Santarém, Portugal rui.jorge@esa.ipsantarem.pt

Histórico do artigo: Recebido a 29 de setembro de 2020 Aceite a 28 de dezembro de 2020

\author{
DIETARY HABITS IN DIFFERENT PANDEMIC PERIODS \\ THROUGHOUT HISTORY: A NARRATIVE REVIEW OF THE \\ LITERATURE
}

Catarina Monteiro'; Rui Jorge ${ }^{1-3^{*}}$

\section{RESUMO}

A quarentena, voluntária ou obrigatória, suscita transformações inevitáveis no estilo de vida. A presente revisão narrativa da literatura visa estudar os hábitos alimentares adotados durante pandemias e epidemias com repercussões mundiais. Enquanto antigamente as doenças infeciosas eram predominantes em indivíduos desnutridos por escassez de alimento, hoje afetam desnutridos devido a hábitos alimentares excessivos e desequilibrados. A Gripe Espanhola originou grande escassez de alimento e insegurança alimentar. A Gripe das Aves e a Gripe A não afetaram hábitos no consumo da carne e ovos, apesar do impacto no setor alimentar. Tanto a Gripe A como a COVID-19 foram exacerbadas pela elevada prevalência de doenças crónicas, nomeadamente a obesidade. A atuação do nutricionista em contexto pandémico ou epidémico, é crucial por promover, junto da população, a adoção de um estilo de vida saudável, principalmente hábitos alimentares, como medida preventiva.

\section{PALAVRAS-CHAVE}

Hábitos alimentares, Pandemia, Quarentena

\section{ABSTRACT}

Quarantine, voluntary or mandatory, causes inevitable changes in lifestyle. This narrative review of literature aims to study the eating habits adopted during pandemics and epidemics with worldwide repercussions. While in the past, infectious diseases were prevalent in individuals malnourished due to food shortages, today they affect malnourished people due to excessive and unbalanced eating habits. The Spanish flu caused great food shortages and food insecurity. Bird flu and influenza A did not affect meat and egg consumption habits, despite the impact on the food sector. Both Influenza A and COVID-19 were exacerbated by the high prevalence of chronic diseases, including obesity. The role of the nutritionist in a pandemic context is crucial for promoting the population to adopt a healthy lifestyle, mainly healthy eating habits, as a preventive measure.

\section{KEYWORDS}

Eating habits, Pandemic, Quarantine

\section{INTRODUÇÃo}

A manifestação de novas doenças transmissíveis e a reemergência de outras que se supunham controladas representam um desafio para a saúde pública. Nestas circunstâncias, diferentes países implementam estritas medidas, que apesar de preventivas, afetam o quotidiano da população. Esta revisão aborda doenças transmissíveis que tiveram repercussões mundiais ao nível do dever de isolamento social, nomeadamente: a Gripe Pneumónica em 1918, a Gripe das Aves em 2003, a Gripe A em 2009 e a doença do coronavírus 2019 (COVID-19) em 2019. Pela ausência de revisões que reflitam os hábitos alimentares adotados por diferentes populações durante os distintos períodos pandémicos, esta revisão visa responder às seguintes questões: Que hábitos alimentares foram adotados em situação de isolamento social? Qual o papel do nutricionista em contexto de emergência de saúde pública?

\section{METODOLOGIA}

Do ponto de vista metodológico procedeu-se à pesquisa nas bases de dados Medline e PubMed sem limitação de data ou país de realização dos estudo, sendo apenas considerados artigos escritos em Português ou em Inglês. As palavras-chave utilizadas foram "pandemic", "quarantine" e "eating habits". Após a leitura dos títulos de todos os artigos obtidos na pesquisa, identificaram-se 61 artigos potencialmente relevantes e após leitura integral (ou em alguns casos apenas do resumo) 22 foram integrados na presente revisão narrativa.

\section{Hábitos Alimentares em Tempo de Pandemia}

Garantir uma alimentação variada e equilibrada é crucial sobretudo perante ocasiões em que a mudança de rotinas é inevitável. Ainda assim, releva-se as condições socioeconómicas no processo saúde-doença. A segurança alimentar é um conceito onde "todas as 
pessoas, em todos os momentos, têm acesso físico e económico a alimentos suficientes, seguros e nutritivos para atender às suas necessidades dietéticas e preferências alimentares para uma vida ativa e saudável" (1). Neste sentido, terão as medidas de afastamento social influenciado os hábitos alimentares a nível global?

\section{Gripe Espanhola (1918-1920)}

A Gripe Espanhola, vulgarmente conhecida como Gripe Pneumónica, é considerada uma das mais mortais da história da humanidade. Estima-se que o número de mortes rondou entre os 17 e os 50 milhões de pessoas (2).

Portugal vivia um período de vulnerabilidade marcado pela pandemia, mas também pela participação na Primeira Guerra Mundial. Era um país predominantemente rural, com condições sociais preocupantes que contribuíram para o aumento da fome, da pobreza e dos conflitos sociais (3). Esta época desencadeou a redução das colheitas e o aumento significativo dos preços dos alimentos, com consequente aumento da desnutrição (4). Mais tarde, o número de infetados pela Gripe Espanhola começou a progredir, as atividades agrícolas foram interrompidas e o impacto da fome rapidamente surgiu (5).

Implicações como a rápida propagação e a alta mortalidade da doença foram favorecidas pelas condições de saúde limitadas, falta de higiene e pela má nutrição. $\mathrm{O}$ aumento da mortalidade entre os 0 e os 4 anos de idade, um grupo tradicionalmente vulnerável, fez com que indivíduos nesta faixa etária recebessem maior atenção por parte da literatura. Julga-se que, pela possibilidade de o bem-estar do feto ser determinado pela saúde da mãe, seja plausível que a infeção no primeiro ou segundo trimestre possa ter aumentado a vulnerabilidade à morte precoce. Do mesmo modo, a saúde precária das mães terá levado à não amamentação dos filhos (6). É possível presumir que existiu maior risco de mortalidade infantil em crianças desnutridas no período pandémico mencionado.

Informações relativas a este período são escassas especialmente em relação à alimentação praticada, mas o regime alimentar era certamente deficiente. $\mathrm{O}$ típico trabalhador rural vivia em condições adversas, alimentava-se mal e consumia água contaminada (quando existia). No entanto, até a década de 1940 era habitual a preparação de receitas caseiras para combater o desconhecido vírus com sintomas semelhantes a uma gripe comum. Por exemplo, para aliviar as dores de garganta mastigava-se gengibre ou pau de alcaçuz, planta onde atualmente se extrai um líquido utilizado em fármacos para a tosse (7). Presume-se ainda que a quantidade de compras reduziu para metade como resultado de comportamentos preventivos, para evitar saídas desnecessárias, por motivos económicos e ainda devido à falta de apetite dos infetados (8).

\section{Gripe das Aves (2003-2004)}

A Gripe das Aves foi uma epidemia que muito se temeu progredir para pandemia. Teve especial enfâse neste artigo pelo consumo vulgar de aves e ovos em Portugal e no mundo (9). Os casos em humanos foram confirmados especialmente em áreas rurais e associados ao contato direto ou indireto com aves infetadas. Contudo, o consumo de aves de capoeira e produtos derivados destes foram confirmados como seguros pela Organização Mundial da Saúde (OMS), desde que sejam asseguradas boas práticas de higiene, incluindo a cozedura adequada (10). O vírus é eliminado pelo calor a uma temperatura de $56^{\circ} \mathrm{C}$ durante 3 horas ou de $60^{\circ} \mathrm{C}$ durante 30 minutos, mas sobrevive durante 4 dias a $22^{\circ} \mathrm{C}$ ou mais de 30 dias a $0^{\circ} \mathrm{C}(10)$.

A epidemia da Gripe Aviária levou ao abate extensivo de aves, porém nem sempre respeitado. O Bangladesh foi dos países mais afetados, mas o consumo de frangos, galinhas e ovos oriundos destas aves foram hábitos mantidos durante a epidemia. As condições onde estes animais habitavam eram também uma problemática regular por não existirem quaisquer regras de higiene. O contacto humano com as aves e inclusive as suas fezes era frequente, o que exacerbava a transmissão do vírus (11). Relativamente aos Europeus, $78,0 \%$ não diminuíram o consumo de carne de aves de capoeira e 83,0\% mantiveram o número de ovos que consumiam. Todavia, quem reduziu o consumo de carne de aves confessou preferir adotar uma atitude cautelosa (12).

Na Turquia, a Gripe Aviária não foi considerada, pela maioria, como uma ameaça à saúde pública, provavelmente pela importância económica do setor, sendo escassas as medidas preventivas (13). Por um lado, pelos mais pobres que realmente precisavam de garantir a criação de animais como forma de sustento familiar e, por outro, as empresas que continuavam a produzir e exportar aves simplesmente por serem processos lucrativos. Este desrespeito das medidas preventivas potenciavam a transmissão do vírus entre animais e humanos.

\section{Gripe A (2009-2010)}

A Gripe Suína, usualmente conhecida como Gripe A, foi a primeira pandemia do século XXI. Existiram pelo menos 18 mil mortes e 491 mil casos confirmados (14). A propagação ocorreu através da transmissão porco-humano.

Apesar da carne de porco ser muito usada na alimentação a nível mundial, verifica-se escassez de estudos sobre o impacto da Gripe A na alimentação. Na Europa, apenas $25,0 \%$ das pessoas declarou ter mudado comportamentos devido à pandemia. Em Portugal, 62,2\% dos cidadãos não alterou quaisquer comportamentos (15).

A evidência sugere que o vírus responsável pela pandemia não seja transmitido pela alimentação (16). Porém, o setor da indústria alimentar sentiu consequências significativas nos mercados da carne de porco. Houve uma queda de vendas, decorrentes do aumento do preço dos produtos para equilibrar os prejuízos do setor (15). Poucos estavam dispostos a adquirir carne de porco, ora pelo preço, ora por precaução. Todavia, garantir a cozedura adequada dos alimentos permite diminuir eventuais riscos de infeção, pelo que era recomendado cozinhar a carne de porco a uma temperatura interna de $70^{\circ} \mathrm{C}(17)$.

Neste cenário pandémico, era primordial promover um estilo de vida saudável que vise garantir a saúde da população a longo prazo. Em oposição, a mortalidade em infetados com Gripe A foi fortemente associada a casos severos de obesidade. Nos Estados Unidos, 94 de 543 doentes morreram infetados com a doença. Destes, 56 tinham um índice de massa corporal (IMC) $\geq 30 \mathrm{~kg} / \mathrm{m}^{2}$ e 28 tinham IMC $\geq 40$ $\mathrm{kg} / \mathrm{m}^{2}$ (61,0\% e 30,0\%, respetivamente). A obesidade de grau III foi a condição clínica mais fortemente associada à mortalidade (18). Doentes infetados e com obesidade tiveram 2 vezes mais probabilidades de serem admitidos ou morrerem em Unidades de Cuidados Intensivos (UCl) (19). Não obstante a acentuada relação da obesidade como uma condição clínica frequente em pacientes com Gripe A, os hábitos alimentares não foram avaliados nem incluídos em nenhum estudo.

\section{COVID-19 (2019 - Presente)}

A COVID-19 foi declarada pela OMS, a 30 de Janeiro de 2020, como uma emergência de saúde pública de interesse internacional (20). A maioria dos países esteve em quarentena, as fronteiras fecharam e todos os eventos públicos foram cancelados. Até janeiro de 2021, o vírus infetou mais de 97 milhões de pessoas e provocou cerca de 2 milhões de mortes em todo o mundo (21). O primeiro caso detetado no mundo ocorreu a 31 de Dezembro de 2019, na China (20).

Em Portugal, o primeiro caso relatado ocorreu a 2 de Março de 
2020 (20) e a 18 de Maio de 2020, foi declarado o primeiro estado de emergência, o recolhimento domiciliário tornou-se obrigatório e sucedeu-se uma nova realidade para todos. Se no passado as doenças crónicas e as doenças infeciosas eram vistas como conceitos independentes, a atual pandemia veio demonstrar que patologias como a obesidade, diabetes e hipertensão arterial podem agravar a manifestação e o tratamento de doenças infeciosas como a COVID-19. Indivíduos com obesidade e expostos à síndrome respiratória aguda grave do coronavírus 2 (SARS-CoV-2) desenvolveram piores prognósticos hospitalares e tiveram maior probabilidade de morrer. A suscetibilidade acrescida ocorre porque indivíduos com excesso de gordura corporal apresentam piores estados de inflamação, logo, a resposta imunitária é tardia e ineficaz (22). No Reino Unido, 72,0\% dos infetados nas UCl tinham excesso de peso ou obesidade (23). Em Itália, 99,0\% dos infetados que morreram tinham doenças crónicas como obesidade, hipertensão arterial, diabetes tipo II, entre outras doenças relacionadas com o estilo de vida (24). Na China, 88,2\% dos indivíduos que morreram com COVID-19 tinham IMC $\geq 25 \mathrm{~kg} / \mathrm{m}^{2}$ (excesso de peso) (25).

Neste seguimento, a Direção-Geral da Saúde (DGS) realizou um inquérito sobre a alimentação dos Portugueses em contexto de contenção social associados à pandemia. Os resultados afirmaram que houve alteração de hábitos alimentares em $45,1 \%$, entre os quais $58,2 \%$ modificaram os hábitos alimentares para melhor e 41,8\% para pior (26). Os motivos associados a tais mudanças foram, sobretudo, alterações do número de idas às compras, alterações do apetite, bem como alteração dos horários de trabalho. Durante a quarentena os Portugueses, de uma forma geral, optaram preferencialmente por bebidas como a água e alimentos como snacks doces, fruta e hortícolas em detrimento de refeições pré-preparadas ou take-away, refrigerantes e bebidas alcoólicas. A diminuição da ingestão de determinados alimentos e o aumento de outros poderá eventualmente esclarecer o facto de $57,3 \%$ dos inquiridos reportar ter mantido o peso corporal durante a quarentena (26).

Apesar das medidas de mitigação garantirem a suficiente disponibilidade de alimentos à população, a insegurança alimentar já era uma realidade anteriormente vivenciada. Existe insegurança alimentar quando o acesso a alimentos nutritivos e suficientes para uma vida ativa e saudável é limitado ou incerto (27). Um estudo relativo aos anos de 2015 e 2016, estimou recorrendo a uma versão adaptada e validada para português da "US Household Food Security Survey Module", uma prevalência de 19,3\% de insegurança alimentar em Portugal (28). Contudo, durante a presente pandemia um estudo realizado pela DGS concluiu que $33,7 \%$ dos inquiridos reportaram preocupação ou incerteza quanto ao acesso aos alimentos por dificuldades económicas e que 8,3\% reportava mesmo dificuldades económicas no acesso aos alimentos (26). Particularizando por regiões do país, o Alentejo foi a região com maior risco de insegurança alimentar (45,0\%), seguida dos Açores (44,5\%), Algarve (41,2\%), Madeira (39,4\%), Lisboa e Norte com a mesma percentagem $(31,8 \%)$ e, por fim, o Centro foi onde existiu menor risco de insegurança alimentar (29,8\%) (26).

Durante o período de isolamento $18,2 \%$ dos Portugueses considerou aderir a um padrão alimentar saudável definido pelo estudo e que incluía o aumento de fruta, hortícolas e pescado. Porém, 10,8\% referiu aderir a um padrão alimentar não saudável, caracterizado pelo aumento de refeições pré-preparadas, snacks salgados, e refrigerantes (26). Quem não conhecia as orientações da DGS na área da alimentação e os que viviam em situação de insegurança alimentar foram os que mais reportaram adotar um padrão de alimentar não saudável (26).

Um estudo conduzido durante a quarentena com base numa amostra de conveniência composta por 3533 participantes Italianos de todas as regiões de Itália concluiu não existirem mudanças quanto aos hábitos alimentares de 46,1\% dos participantes (29). Dos que reportaram alterar os seus hábitos alimentares, 16,7\% reporta ter mudado para melhor e 37,2\% para pior. Mais de metade sentiu diferenças na perceção de fome ou saciedade, sendo que a maioria reportou ter mais apetite. A perceção de aumento do peso corporal foi observada em 48,6\% (29).

\section{ANÁLISE CRÍTICA}

\section{Gripe Espanhola (1918-1920)}

A falta de consenso a respeito da etiologia da doença e dos meios medicinais para a combater, contribuíram para que a população adotasse estratégias de autodefesa apenas com auxílio do que a natureza disponibilizava (7). O regime alimentar era nutricionalmente deficiente nos continentes em desenvolvimento e a inacessibilidade de alimentos, por escassez ou falta de dinheiro, foi sentida à escala mundial. No continente Africano, teve especial enfâse porque as repercussões na produção, transporte e fornecimento de alimentos à população foram sentidas mesmo após a pandemia ter terminado.

Em 1918 a grande preocupação era garantir alimento. A não existência de acesso regular a alimentos em quantidade e qualidade agravou o bem-estar das famílias. A pobreza gerou insegurança alimentar e desnutrição, a saúde ficou debilitada o que impossibilitou ter capacidade de trabalhar, ocasionando novamente pobreza. Este círculo vicioso perpetuou e intensificou a insegurança alimentar que, por conseguinte, é implicativa no aparecimento e o desenvolvimento pandemias e epidemias. Nestas condições, a rápida transmissão do vírus foi exacerbada pela desnutrição e pelas carências alimentares e as consequências alusivas à má nutrição persistiram durante esta época, mas também nas gerações seguintes.

São estes momentos pandémicos em que todos lutam pela equidade em saúde, que se percebe o impacto das desigualdades socioeconómicas. Neste aspeto, as emergentes áreas de atuação da profissão de nutricionista são crucias. No passado as recomendações nutricionais advinham do médico que habitava na aldeia. Atualmente existem também nutricionistas na área clínica, importantes no processo saúde-doença e ainda nutricionistas na área comunitária que têm em consideração a saúde pública através da promoção e avaliação do consumo alimentar populacional. As diferentes áreas de desempenho profissional do nutricionista podem levar a um melhor estado nutricional e favorecer o prognóstico clínico, diminuindo o número de mortes.

\section{Gripe das Aves (2003-2004)}

Apesar de uma epidemia maioritariamente virulenta em aves domésticas, animal vulgarmente consumido por todos, os hábitos alimentares implementados durante a Gripe Aviária foram pouco modificados. Apenas 16,0\% dos cidadãos Europeus diminuíram o consumo de carne de aves e produtos derivados em relação aos 6 meses anteriores. Os restantes pensavam não existir qualquer tipo de risco envolvido no consumo de aves ou ovos (11).

Estes dados, verdadeiramente alarmantes, requerem mais estudos que tencionem compreender as atitudes da população neste período tão incerto. Embora a criação de aves forneça apoio essencial para a subsistência das famílias, este sustento é arriscado durante a situação vivida, por aumentar o risco de contágio, devido ao contato próximo com as aves. No entanto, uma das possíveis explicações pode ser a escassa literacia em saúde sobre as práticas de higiene a adotar. Por um lado, proveniente da população em geral devido à falta de conhecimentos sobre a manipulação adequada e o consumo seguro dos animais, por outro lado, por parte dos produtores por não aplicarem diariamente 
princípios de higiene e segurança alimentar no trabalho.

Apesar de as doenças transmitidas pela carne terem diminuído com as alterações dos sistemas de produção da carne, a permanência do problema tem sido ilustrada nos últimos anos. Assim, a garantia de boas práticas de higiene e segurança alimentar ou a aplicação de procedimentos normativos associados à restauração são funções do nutricionista, e particularmente importantes na génese de uma epidemia como a Gripe das Aves, por evitarem a manuseamento e o consumo de alimentos impróprios e perigosos para a saúde.

\section{Gripe A (2009-2010)}

A transição da génese e propagação das doenças infeciosas ocorreu na pandemia da Gripe A. Se as pandemias anteriores eram provenientes das condições sanitárias deficientes, a partir da pandemia de 2009 a presença de doenças crónicas em doentes infetados passou a ser das principais causas de morte. A relação entre doentes infetados com o vírus da Gripe A e a obesidade foi clara. Nenhum estudo pretendeu avaliar os hábitos alimentares adotados durante a quarenta, possivelmente porque a quarenta foi unicamente obrigatória para doentes infetados, sendo que parte destes estavam hospitalizados. Todavia, a elevada prevalência de indivíduos com obesidade, que foram infetados, pode também refletir que o estilo de vida adotado por uma parte relevante da população não era o preconizado.

A adoção de comportamentos saudáveis, o trabalho dos profissionais de saúde e em particular dos nutricionistas torna-se cada vez mais preeminente à medida que se verificam novas vagas infeciosas. Promover um melhor estado nutricional da população e uma substancial redução na prevalência de excesso de peso e obesidade reduziria o número de internamentos, a sobrecarga dos hospitais e as mortes de doentes infetados com Gripe A.

\section{COVID-19 (2019 - Presente)}

Pela singularidade da emergência de saúde pública e pela crescente necessidade em entender e dominar o novo coronavírus, estudos com variados propósitos têm vindo a ser publicados. No que diz respeito à alimentação, em Portugal verificou-se uma maior procura pela adoção de hábitos alimentares saudáveis. Esta preocupação pode ser esclarecida pela constante permanência em casa e a usual exposição a momentos familiares, existindo mais tempo para planear o que comer. Eventualmente devido ao aumento do food craving que se refere ao intenso desejo de consumir um determinado alimento (30). Assim, o emocional anseio e a situação difícil que se vive motiva a alterações nas escolhas e porções alimentares em resposta à ansiedade e ao tédio (31). Desta forma, confirma-se que as limitações mundiais necessárias para conter o agente infecioso afetaram rotinas e hábitos alimentares. A dicotomia das doenças crónicas e das doenças agudas infeciosas tem vindo a preocupar os profissionais de saúde, pois estão cada vez mais a exprimir-se conjuntamente. Indivíduos com obesidade e piores estados nutricionais são mais suscetíveis a desenvolver complicações quando infetados. Se no passado as doenças crónicas e as doenças agudas infeciosas eram consideradas conceitos distintos e sem qualquer associação, a mais recente pandemia veio demonstrar que doenças crónicas como a obesidade, a hipertensão arterial e a diabetes podem agravar o prognóstico clínico durante a de infeção por COVID-19 (32).

Desde a Gripe Espanhola que a malnutrição é associada a piores prognósticos hospitalares. Este tópico obriga à reflexão dos determinantes das doenças infeciosas, visto que antigamente eram predominantes em indivíduos desnutridos por escassez de alimento e hoje afetam desnutridos devido a hábitos alimentares excessivos e desequilibrados. Assim, a atuação do nutricionista é irrefutável, até em contexto pandémico, por ser um profissional de saúde com competências que permitem promover e incentivar a população a adotar um estilo de vida saudável como medida preventiva.

\section{CONCLUSÕES}

A presente revisão reforça a importância da intervenção do nutricionista na promoção da saúde e prevenção da doença. Promover cuidados de higiene em produção animal, promover a redução das prevalências das principais doenças crónicas não transmissíveis e garantir um bom estado nutricional da população pode prevenir o surgimento de novas pandemias e, sobretudo em contexto pandémico, contribuir para a redução de internamentos hospitalares e do número de mortes. Tais estratégias devem ser instruídas por nutricionistas e suportada por diversos profissionais da área alimentar e da saúde, para que em futuras pandemias a população esteja mais preparada e consciente dos comportamentos a adotar do ponto de vista alimentar.

\section{REFERÊNCIAS BIBLIOGRÁFICAS}

1. FAO. (2008). An Introduction to the Basic Concepts of Food Security.

2. OMS. (2018). Pandemic influenza: an evolving challenge. Consultado a 2 de Setembro de 2020 em: https://www.who.int/influenza/pandemic-influenza-an-evolvingchallenge/en/.

3. David de Morais, J. A. (2007). Tifo epidémico em Portugal: um contributo para o seu conhecimento histórico e epidemiológico. Medicine History, 15(3), 291-307.

4. Humphreys, M. (2018). The influenza of 1918: Evolutionary perspectives in a historical context. Evolution, Medicine and Public Health, 2018(1), 219-229.

5. Schoenbaum, S. C. (2001). The impact of pandemic influenza, with special reference to 1918. International Congress Series, 1219(C), 43-51.

6. Reid, A. (2005). The Effects of the 1918-1919 Influenza Pandemic on Infant and Child Health in Derbyshire. Medical History, 49(1), 29-54.

7. Souza, C. M. C. (2009). A Gripe Espanhola na Bahia: Saúde, política e medicina em tempos de epidemia. Fiocruz.

8. Wren-Lewis, S., \& Keogh-Brown, M. (2009). The possible macroeconomic impact on the UK of an influenza pandemic. Economics Series Working Papers.

9. OMS. (2020). Cumulative number of confirmed human cases for avian influenza A(H5N1), 2003-2020. Consultado a 2 de Setembro de 2020 em: https://www.who. int/influenza/human_animal_interface/2020_10_07_tableH5N1.p df?ua=1.

10. OMS. (2012). H5N1 influenza. Consultado a 1 de Julho de 2020 em: https://www. who.int/news-room/q-a-detail/h5n1-influenza.

11. Comissão Europeia. (2006). Special Eurobarometer: Avian Influenza. Consultado a 18 de Junho de 2020 em: https://ec.europa.eu/commfrontoffice/publicopinion/ archives/ebs/ebs_257_en.pdf.

12. Sultana, R., Nahar, N., Rimi, N. A., Azad, S., Islam, M. S., Gurley, E. S., \& Luby, S. P. (2012). Backyard poultry raising in Bangladesh: a valued resource for the villagers and a setting for zoonotic transmission of avian influenza. A qualitative study. Rural and Remote Health, 12(197), 1927.

13. Edirne, T., Avci, D. K., Dagkara, B., \& Aslan, M. (2011). Knowledge and anticipated attitudes of the community about bird flu outbreak in Turkey, 2007-2008: a surveybased descriptive study. International Journal of Public Health, 56(2), 163-168.

14. OMS. (2010). Pandemic (H1N1) 2009. Consultado a 3 de Setembro de 2020 em: https://www.who.int/csr/don/2010_08_06/en/.

15. Comissão Europeia. (2009). Flash Eurobarometer 287-Influenza H1N1: Analytical Report. Consultado a 26 de Janeiro de 2021 em: https://ec.europa.eu/commfrontoffice/ publicopinion/flash/fl_287_en.pdf.

16. EFSA. (2010). Statement on Food safety considerations of novel H1N1 influenza virus infections in humans. EFSA Journal, 8(6), 1629.

17. United States Department of Agriculture. (2011). Kitchen Thermometers. https:// www.fsis.usda.gov/wps/wcm/connect/d8151061-bb50-46db-b87e-a3b9022c0c56/ Kitchen_Thermometers.pdf?MOD=AJPERES. 
18. Louie, J. K., Acosta, M., Samuel, M. C., Schechter, R., Vugia, D. J., Harriman, K., \& Matyas, B. T. (2011). A Novel Risk Factor for a Novel Virus: Obesity and 2009 Pandemic Influenza A (H1N1). Clinical Infectious Diseases, 52(3), 301-312.

19. Fezeu, L., Julia, C., Henegar, A., Bitu, J., Hu, F. B., Grobbee, D. E., Kengne, A.P., Hercberg, S., \& Czernichow, S. (2011). Obesity is associated with higher risk of intensive care unit admission and death in influenza $A(H 1 N 1)$ patients: a systematic review and meta-analysis. Obesity Reviews, 12(8), 653-659.

20. OMS. (2020b). Archived: WHO timeline-covid-19. Consultado a 25 de Janeiro de 2020 em: https://www.who.int/news/item/27-04-2020-who-timeline---covid-19.

21. OMS. (2020c). WHO Coronavirus Disease (COVID-19) Dashboard. Consultado a 25 de Janeiro de 2021 em: https://covid19.who.int/.

22. Malavazos, A. E., Corsi Romanelli, M. M., Bandera, F., \& lacobellis, G. (2020). Targeting the Adipose Tissue in COVID-19. Obesity, 28(7), 1178-1179.

23. Mahase, E. (2020). Covid-19: most patients require mechanical ventilation in first 24 hours of critical care. BMJ, 368, m1201.

24. Italian National Institute of Health. (2020). Characteristics of COVID-19 patients dying in Italy on April 2nd, 2020. https://www.epicentro.iss.it/en/coronavirus/bollettino/ Report-COVID-2019_2_april_2020.pdf.

25. Peng, Y. D., Meng, K., Guan, H. Q., Leng, L., Zhu, R. R., Wang, B. Y., He, M. A., Cheng, L. X., Huang, K., \& Zeng, Q. T. (2020). Clinical characteristics and outcomes of 112 cardiovascular disease patients infected by 2019-nCoV. ZHonghua Journal of Cardiovascular Diseases, 48(6), 450-455.

26. DGS. (2020). REACT-COVID: Inquérito sobre alimentação e atividade física em contexto de contenção social. Consultado a 26 de janeiro de 2021 em: https://www. dgs.pt/programa-nacional-para-a-promocao-da-atvidade-fisica/ficheiros-externospnpaf/rel_resultados-survey-covid-19-pdf.aspx.

27. Pooler, J. A., Hartline-Grafton, H., DeBor, M., Sudore, R. L., \& Seligman, H. K. (2019). Food Insecurity: A Key Social Determinant of Health for Older Adults. In Journal of the American Geriatrics Society, 67(3), 421-424.

28. Gregório, M. J., Rodrigues, A. M., Graça, P., de Sousa, R. D., Dias, S. S., Branco, J. C., \& Canhão, H. (2018). Food Insecurity Is Associated with Low Adherence to the Mediterranean Diet and Adverse Health Conditions in Portuguese Adults. Frontiers in Public Health, 6(38).

29. Di Renzo, L., Gualtieri, P., Pivari, F., Soldati, L., Attinà, A., Cinelli, G., Leggeri, C., Caparello, G., Barrea, L., Scerbo, F., Esposito, E., \& De Lorenzo, A. (2020). Eating habits and lifestyle changes during COVID-19 lockdown: an Italian survey. J Transl Med, 18, 229.

30. Rodríguez-Martín, B. C., \& Meule, A. (2015). Food craving: new contributions on its assessment, moderators, and consequences. Frontiers in Psychology, 6.

31. Moynihan, A. B., Tilburg, W. A. P. van, Igou, E. R., Wisman, A., Donnelly, A. E., \& Mulcaire, J. B. (2015). Eaten up by boredom: consuming food to escape awareness of the bored self. Frontiers in Psychology, 6(APR), 369.

32. Yang, J., Zheng, Y., Gou, X., Pu, K., Chen, Z., Guo, Q., Ji, R., Wang, H., Wang, Y., \& Zhou, Y. (2020). Prevalence of comorbidities and its effects in patients infected with SARS-CoV-2: a systematic review and meta-analysis. International Journal of Infectious Diseases, 94, 91-95. 